\section{An ode to polyethylene - ADDENDUM}

doi: https://doi.org/10.1557/mre.2019.15

The following footnote should be included in this article [1]:

This paper was commissioned for publication by David Cahen, who served as Editor-in-Chief of this journal from 2014-2018.
Svetlana V. Boriskina

REFERENCE:

1. Boriskina S. V., An ode to polyethylene. MRS Energy \& Sustainability, 6, E14. (2019). 